Ebisu Ebisu

Études japonaises Études japonaises

47 | printemps-été 2012

Catastrophes du 11 mars 2011, désastre de

Fukushima : fractures et émergences

\title{
La reconstruction de la commune d'ōtsuchi : étude des souhaits des habitants
}

The Reconstruction of Ōtsuchi: a Study Based on the Will of Local Residents 東日本大震桨の大槌町の復駧に関して一住民の望みに関する研究

Kumi Eguchi, Aya Kubota, Setsuji Nagase et Takefumi Kurose

\section{OpenEdition \\ Journals}

Édition électronique

URL : http://journals.openedition.org/ebisu/416

DOI : 10.4000/ebisu.416

ISSN : 2189-1893

Éditeur :

Institut français de recherche sur le Japon (UMIFRE 19 MAEE-CNRS), Maison franco-japonaise

Édition imprimée

Date de publication : 1 juin 2012

Pagination : 175-187

ISSN : 1340-3656

Référence électronique

Kumi Eguchi, Aya Kubota, Setsuji Nagase et Takefumi Kurose, « La reconstruction de la commune d'Ōtsuchi : étude des souhaits des habitants », Ebisu [En ligne], 47 | printemps-été 2012, mis en ligne le 17 novembre 2014, consulté le 02 mai 2019. URL : http://journals.openedition.org/ebisu/416 ; DOI 10.4000/ebisu.416 


\title{
La reconstruction de la commune d'Ōtsuchi Étude des souhaits des habitants
}

\author{
EGUCHI Kumi 江口久美, KUBOTA Aya 窪田亜矢, \\ NAGASE Setsuji 永瀬節治, KUROSE Takefumi 黒瀬武史
}

La commune d'Ōtsuchi 大槌町 a été détruite à plus de $60 \%$ par le tremblement de terre et le tsunami de 2011. Cet article relate un travail réalisé par le laboratoire de design urbain de l'université de Tokyo, en coopération avec les habitants du quartier d'Akahama 赤浜, pour proposer un plan de reconstruction qui prenne en compte l'histoire des désastres et les attentes des résidents.

\section{Préliminaires}

La commune d'Ōtsuchi est une ville littorale située sur la côte Pacifique dans le département d'Iwate 岩手. D’une superficie de $201 \mathrm{~km}^{2}$ et d'une

\footnotetext{
V Eguchi Kumi est chercheure invitée, UMR CNRS LAVUE 7218 (Laboratoire architecture, ville, urbanisme, environnement), équipe AUS (Architecture, urbanisme et société). Kubota Aya est maître de conférences au département d'ingénierie urbaine, faculté d'ingénierie, université de Tokyo ; président du Conseil du paysage de la commune d'Urayasu (Urayasu-shi Keikan shingikai 浦安市景観審議会), membre du Conseil environnemental du département de Tokyo (Tōkyō-to Kankyō shingikai 東京都環境審 議会). Nagase Setsuji est maître assistant en système de conservation urbaine, Research Center for Advanced Science and Technology, université de Tokyo, lauréat 2010 du prix Yuasa 湯浅賞 de soutien à la recherche (prix de thèse de doctorat). Kurose Takefumi est ingénieur, maître assistant au département d'ingénierie urbaine, faculté d'ingénierie, université de Tokyo. Lauréat 2006 du prix du Doyen de la faculté d'ingénierie.
} 
population en 2010 d'environ 15000 habitants, l'essentiel de l'activité d'Ōtsuchi tourne autour de l'industrie de la pêche. La commune est le fruit du rassemblement de plusieurs villages de pêcheurs dont le centre se situe à l'embouchure des rivières d'Ōtsuchi et de Kozuchi 小錧, près de la gare ferroviaire d'Ōtsuchi. La ville est également composée des quartiers moins peuplés d'Ando 安渡 et d'Akahama, qui se situent sur la côte nord de la baie d'Ōtsuchi, et du quartier de Kirikiri 吉里吉里 qui fait face à la baie de Funakoshi 船越 (fig. 1). La " commune» est l'ensemble des communautés des petits villages indépendants et de la communauté de la ville du centre.

Cet article s'intéresse plus particulièrement au quartier d'Akahama qui comptait 863 habitants en 2010. En 1973, s’y était installé le Centre de recherche sur la mer d'Ōtsuchi, affilié au centre de recherche sur l'atmosphère et l'océan de l'université de Tokyo (Ōtsuchi Marine Research Center of the Atmosphere and Ocean Research Institute of Tokyo University, Tōkyō

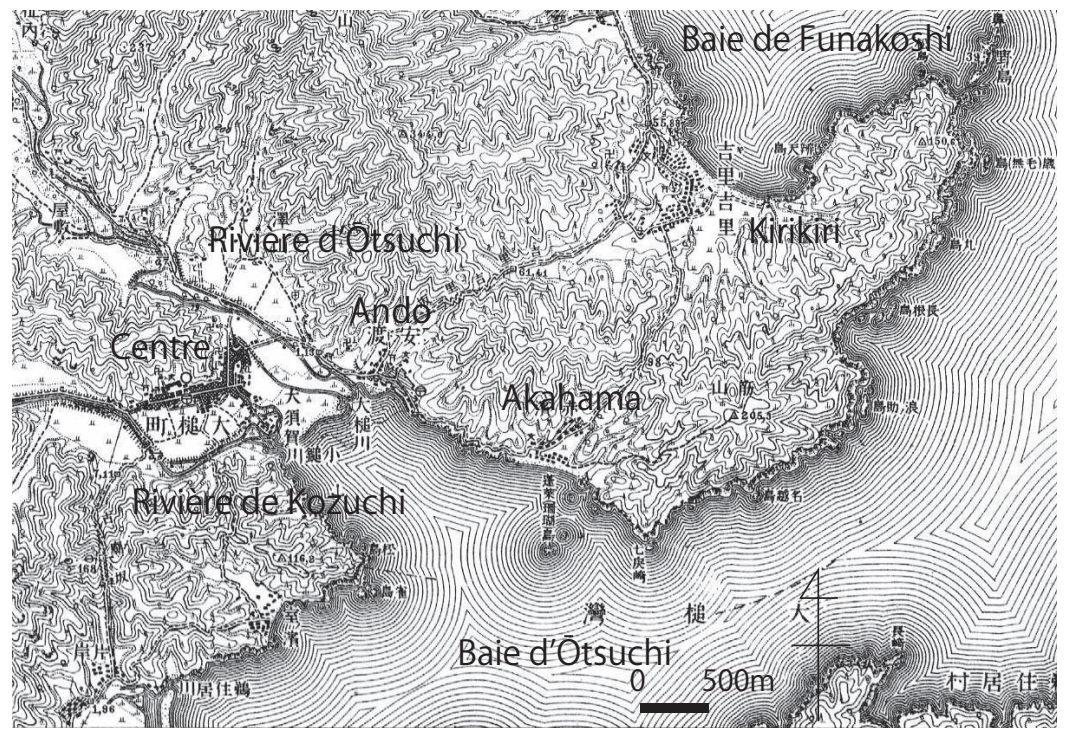

Fig. 1 Ville d'Ōtsuchi en 1916 (1:50 000). Extrait de Taishō go nen sokuzu 大正五年測図 (Carte de l'an 5 de l'ère Taishō [1916]), établie par Dai-Nihon teikoku Rikuchi sokuryōbu 大日本帝 国陸地測量部 (Département de topographie de l'Armée de terre du Grand Japon impérial), modifié par les auteurs. 
daigaku kaiyō kenkyūjo fuzoku Ōtsuchi rinkai kenkyū sentā 東京大学海洋 研究所付属大桘臨海研究センター). La coopérative locale des pêcheurs avait bien accueilli la venue de ce centre et avait partagé les droits de pêche avec lui. Après le tremblement de terre du 11 mars 2011, l'université de Tokyo a créé un Centre de soutien à la reconstruction (Tōkyō daigaku fukkō shien shitsu 東京大学復興支援室) et de nombreux laboratoires du centre ont participé, selon leurs capacités, à des opérations de soutien dans diverses zones endommagées. Notre laboratoire de design urbain s'est rapproché des habitants d'Akahama pour la reconstruction.

\section{Histoire des désastres et état des lieux des dommages dus au dernier séisme}

Si l'on remonte à la fin du Xix ${ }^{\mathrm{e}}$ siècle, Akahama a subi le tsunami de Meiji Sanriku 明治三陸津波 en 1896 (an 29 de l'ère Meiji). La hauteur maximum de la vague y avait été de 3,80 m, 500 maisons avaient été détruites faisant 500 morts. La commune d'Ōtsuchi avait été reconstruite sur place.

En 1933 (an 8 de l'ère Shōwa), le tsunami de Shōwa Sanriku 昭和三陸津 波 a amené une vague de 4,75 m de haut à Akahama, détruisant 480 maisons et faisant 47 morts dans la commune d'Ōtsuchi (Meiji daigaku 2011). Cependant, le centre de la commune d'Ōtsuchi, qui était le centre économique de la région, avait adopté un plan de reconstruction sur place avec l'édification d'une digue brise-lame (bōrō tei 防浪堤) le long du littoral entre les deux rivières. Par ailleurs, les quartiers d'Akahama et d'Ando avaient fait l'objet d'un projet de reconstruction autour d'un complexe de routes combinant une route parallèle à la côte et une route montant vers la montagne. En outre, 20 maisons du quartier d'Ando avaient été déménagées pour être reconstruites sur un terrain plus élevé à une hauteur de $8 \mathrm{~m}$.

En 1960 (an 35 de l'ère Shōwa), le séisme du Chili チリ地震 a provoqué un tsunami d'une hauteur de 3,90 m dans le centre de la commune d'Ōtsuchi et 267 maisons furent détruites sur l'ensemble de la commune. Cependant, il n'y eut pas de morts grâce à la digue et au déplacement des maisons sur les hauteurs qui avait été organisé après les tsunamis précédents. À la suite du tsunami du Chili, un nouveau projet d'aménagement de la ville prévoyait pour le quartier d'Ando le déplacement des maisons sur 


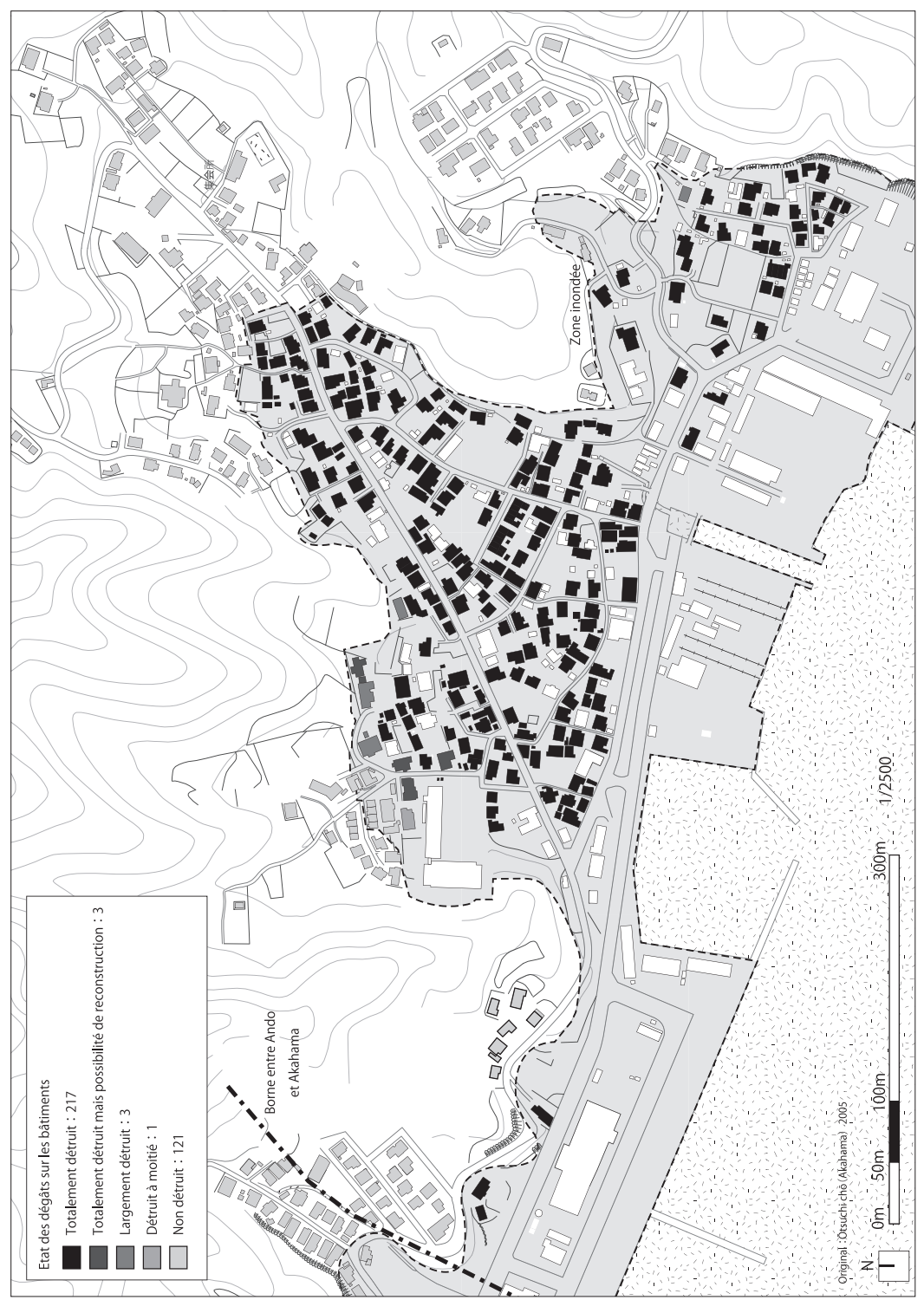

Fig. 2 Akahama : zone touchée par le tsunami. 
les hauteurs. Une nouvelle digue longue de $1263 \mathrm{~m}$ fut construite dans la baie d'Ōtsuchi (Toshi keikaku isan kenkyū kai 2011).

Mais le tsunami qui est survenu en 2011 était plus haut que les précédents. La hauteur de l'inondation par le tsunami était de 12,9 m à Akahama. La plus grande partie du quartier résidentiel d'Akahama et des installations du port ont été sérieusement endommagées (fig. 2). Le tremblement de terre a tué 107 habitants. Combiné à l'exode rural qui existait déjà auparavant, on prévoit que la commune pourrait perdre plus de la moitié de sa population. Près de $60 \%$ des maisons ont été détruites par le tsunami et par les incendies survenus après le tremblement de terre. La photo du bateau croiseur à cabine Hamayuri はまゆり, comme posé sur le toit de l'auberge Akabu あか ぶ à Akahama, a fait le tour du monde comme symbole de l'ampleur de la vague dévastatrice qui a touché le Japon (fig. 3).

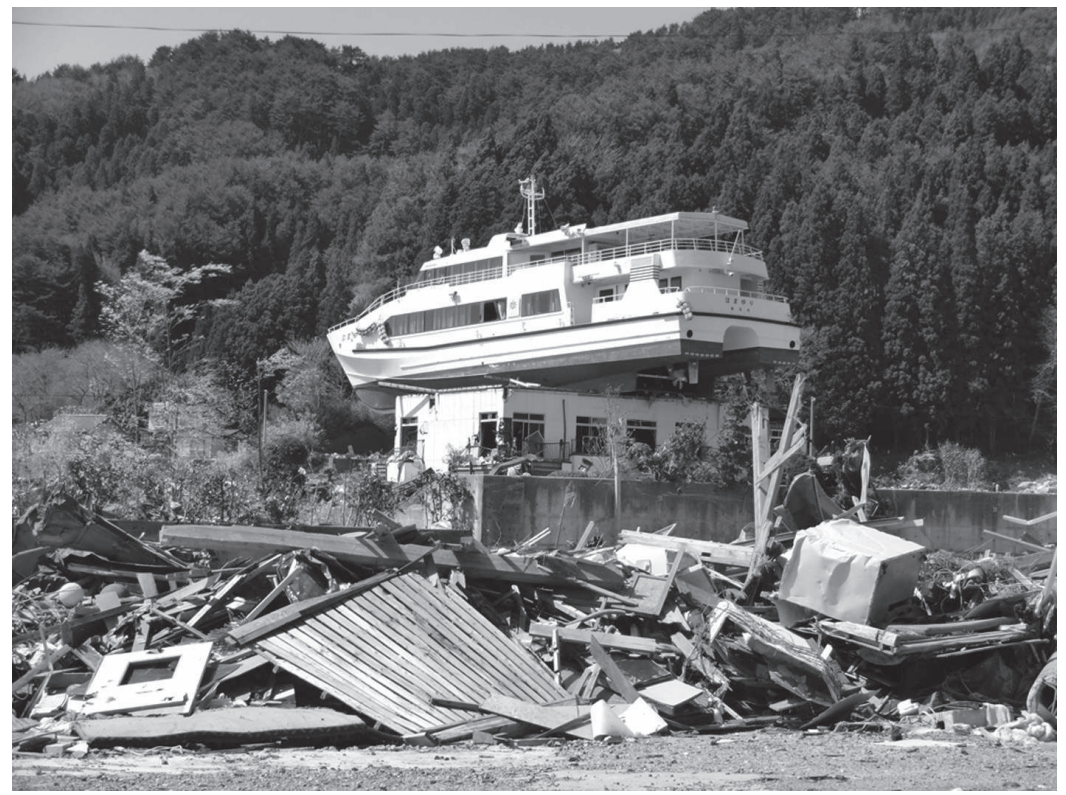

Fig. 3 Le bateau Hamayuri sur l'auberge Akabu. 


\section{Les souhaits et les orientations exprimés par les habitants}

En juin, notre équipe a interviewé une vingtaine d'habitants du quartier d'Akahama pour recueillir leurs opinions concernant la reconstruction. Nous avons tout d'abord noté leur sentiment de ne pas être suffisamment consultés par les autorités locales pour discuter d'un possible projet de reconstruction. De fait, à la suite du tremblement de terre, l'administration fonctionnait difficilement en raison du décès du maire et de nombreux fonctionnaires, et n'a pas été en état, avant une période relative longue, de préparer un projet concret de reconstruction. Les habitants étaient mécontents de voir l'administration ne consulter qu'une partie d'entre eux. Ainsi, en avril, le bateau Hamayuri a été démonté sans qu'il y ait eu, selon les habitants, de discussion commune au préalable.

Nous avons commencé un travail d'enquête pour comprendre les attentes en assistant à des réunions publiques et en consultant une personne devenue clé dans la communauté. L'administration a organisé une discussion avec les habitants le 17 juin. Toutefois, il ne s'est agi que de présenter des orientations générales pour un plan de reconstruction établi d'après le plan modèle transmis par le département et sans donner de précisions ou de plan détaillé. Voici quelques réactions des habitants que l'on a pu noter lors de cette réunion : "Nous souhaitons un urbanisme participatif par les habitants pour les habitants. " "Nous voulons que l'administration organise un urbanisme participatif avec le quartier et en créant une commission autonome. " " Nous voudrions que l'administration crée un plan qui respecte le caractère de chaque village."

C'est dans ce contexte que M. Kanda Yoshinobu 神田義信, directeur de l'antenne d'Akahama du Centre culturel de la commune d'Ōtsuchi (Ōtsuchi-chō chūō kōminkan Akahama bunkan 大槌町中央公民館赤浜分 館) a exposé sa vision pour l'avenir d'Akahama. Il s'agit d'une personne clé de ce quartier qui réfléchit avec inquiétude à l'avenir de la communauté. Étant donné la situation difficile, les habitants n'avaient pas toujours le temps, la patience ou l'envie de discuter de l'avenir. M. Kanda a eu un rôle moteur pour promouvoir cette réflexion.

Bien que M. Kanda n'ait pas à ce moment toutes les informations techniques en main pour proposer un plan de reconstruction précis, le souci est de faire avancer la réflexion. En particulier, il ne savait pas encore quelle 
hauteur de digue serait nécessaire, quels espaces resteraient inondables ou seraient protégés. Au cour de la proposition de M. Kanda se trouve la volonté de déménager toutes les habitations sur les hauteurs afin que la population vive en sécurité. Un habitant ayant souligné que le centre d'Akahama, cœur de la vie de la commune, était situé dans la rue où avait été retrouvé le Hamayuri, il a été proposé de créer deux niveaux de construction afin de préserver la fonction de ce lieu : un quartier résidentiel à une hauteur de 5 à 6 mètres, en creusant dans la montagne à l'est d'Akahama, et un quartier d'activités dans la zone inondée qui serait abrité par une digue de $10 \mathrm{~m}$ de haut. Plusieurs routes d'évacuation seraient aménagées pour quitter rapidement le quartier d'activités si nécessaire. La proposition prévoyait aussi une route vers Kirikiri pour maintenir la relation qui avait toujours été forte entre Akahama et ce village. En ce qui concerne les restes du bateau Hamayuri, il était projeté d'en conserver la mémoire sur place. Quant à l'école primaire d'Akahama, la proposition était de la déménager et de réunir ailleurs les écoles de la commune, mais de transformer le site en centre communautaire équipé de manière à pouvoir servir de refuge en cas de nécessité.

Cette proposition insiste aussi sur les questions des logements publics et de l'emploi. La création de logements publics a pour objectif d'étendre les possibilités de choix de vie et d'accueillir des personnes venant d'ailleurs. Par ailleurs, la disparition des installations portuaires pour la pêche a occasionné une importante perte d'emplois. M. O., pêcheur à Akahama, a exprimé le désir qu'on remette en état les installations de pêche comme avant le séisme et de lancer la reconstruction indépendamment du soutien national. Pour cela, M. Kanda proposait que la pêche soit rendue possible tout au long de l'année (pour le moment la culture des algues empêche de pêcher la moitié de l'année, ce qui signifie aussi pour les pêcheurs des revenus limités à quelques mois de l'année). Il proposait également la création d'emplois grâce à l'implantation d'entreprises et grâce à la labellisation d'une marque d'Ōtsuchi afin de promouvoir la vente des produits locaux.

Le dernier point que nous souhaitons souligner est la nécessaire mise en place d'une organisation locale pour les habitants d'Akahama afin de réfléchir à la vie du quartier. 


\section{Proposition de plan pour la reconstruction d'Akahama par notre laboratoire}

\section{Les clés pour la planification}

Deux éléments principaux doivent orienter la planification. Premièrement l'utilisation des terrains doit être fondée sur un plan de prévention contre les désastres. Deuxièmement, il nous faut aussi prendre en compte la chute de la population locale. À la suite du séisme, le nombre de foyers a diminué de 300 à 100 .

\section{Quatre zones d'occupation des terrains}

Lidentité future d'Akahama peut être exprimée par la formule suivante : "Une ville littorale tranquille qui reçoit les richesses de l'océan (Umi no yutakasa o kyōju shinagara, odayaka ni sumitsugu machi 海の豊かさを享受し ながら、穏やかに住み継ぐまち). Nous avons différencié quatre zones d'utilisation des terrains (A, B, C, D) (fig. 4) :

- A : zone littorale/zone inondable en avant de la digue ;

- B : zone inférieure inondable en cas de grand tsunami située entre deux lignes de protection (une digue et une route sur remblais) ;

- C : zones non inondables à créer par creusement de la montagne ou par remblais ;

- D : zones en hauteurs non inondables existantes ou à étendre.

\section{Deux lignes de protection contre la mer}

La route principale, la route d'Akahama (Akahama kaidō 赤浜街道), a été inondée par le tsunami. Or cette route a une signification historique, elle est la structure principale du quartier. Nous envisageons de la conserver en la surélevant sur un remblai (morido 盛土). Ce remblai sert ainsi de deuxième ligne pour la prévention des désastres en plus d'une digue en bordure de la mer.

\section{Les objectifs finaux}

Le premier point est d'assurer la sécurité des résidences pour que les habitants n'aient pas à s'échapper en cas d'un tsunami similaire à celui de 2011. Les pavillons seront construits en zone non-inondée et les logements collectifs seront construits dans des zones où le niveau du sol sera 
suffisamment surélevé pour ne pas être atteint par un tsunami, comme cela avait été proposé par les habitants afin de préserver l'animation au centre du quartier, le long de la route d'Akahama.

Le deuxième point est d'assurer la sécurité du réseau routier. Nous proposons une structure urbaine simple et rationnelle afin de pouvoir, en cas de nécessité, se mettre rapidement à l'abri en utilisant les nombreuses routes d'évacuation. Un réseau de routes doit relier les villages entre eux. Dans Akahama, la route se composera de l'axe principal (la route d'Akahama surélevée), d'une route d'évacuation depuis la zone inférieure vers la route d'Akahama, d'une route d'évacuation grimpant dans la montagne et d'une route de contournement au pied de la montagne.

Le troisième point est la construction d'un lieu de souvenir et d'étude pour les enfants. Nous proposons d'une part d'aménager un parc commémoratif pour les victimes du désastre à l'endroit où se trouvait le bateau Hamayuri, d'autre part de dédier un espace à l'étude du processus de revalorisation de la zone littorale par la restauration de son paysage naturel et de sa plage de sable.

Le quatrième point est de promouvoir une certaine autonomie locale et l'intégration de sources d'énergies propres. Nous envisageons à cet effet l'installation de panneaux solaires et de batteries de stockage, la création de puits, l'aménagement de sites agricoles et la construction d'entrepôts de stockage alimentaire. Enfin nous prévoyons, comme cela était proposé par les habitants, d'aménager un espace communautaire pour la prévention des désastres sur le site actuel de l'école primaire d'Akahama.

Le cinquième point, qui s'inspire encore une fois des demandes des habitants, est un projet de " pêcherie créative " (sōzō teki na gyogyō 創造的な漁 業). Nous proposons de commencer la construction de nouvelles installations pour la pêche sans attendre les soutiens du département et de la Coopérative locale des pêcheurs. Ce projet consiste ensuite à accueillir de nouvelles ressources humaines.

\section{En guise de conclusion}

Des bénévoles d'Akahama ont lancé une Association de réflexion sur la reconstruction d'Akahama (Akahama no fukkō o kangaeru kai 赤浜の復興 を考える会) le 10 août, à peu près au moment ou nous présentions notre 


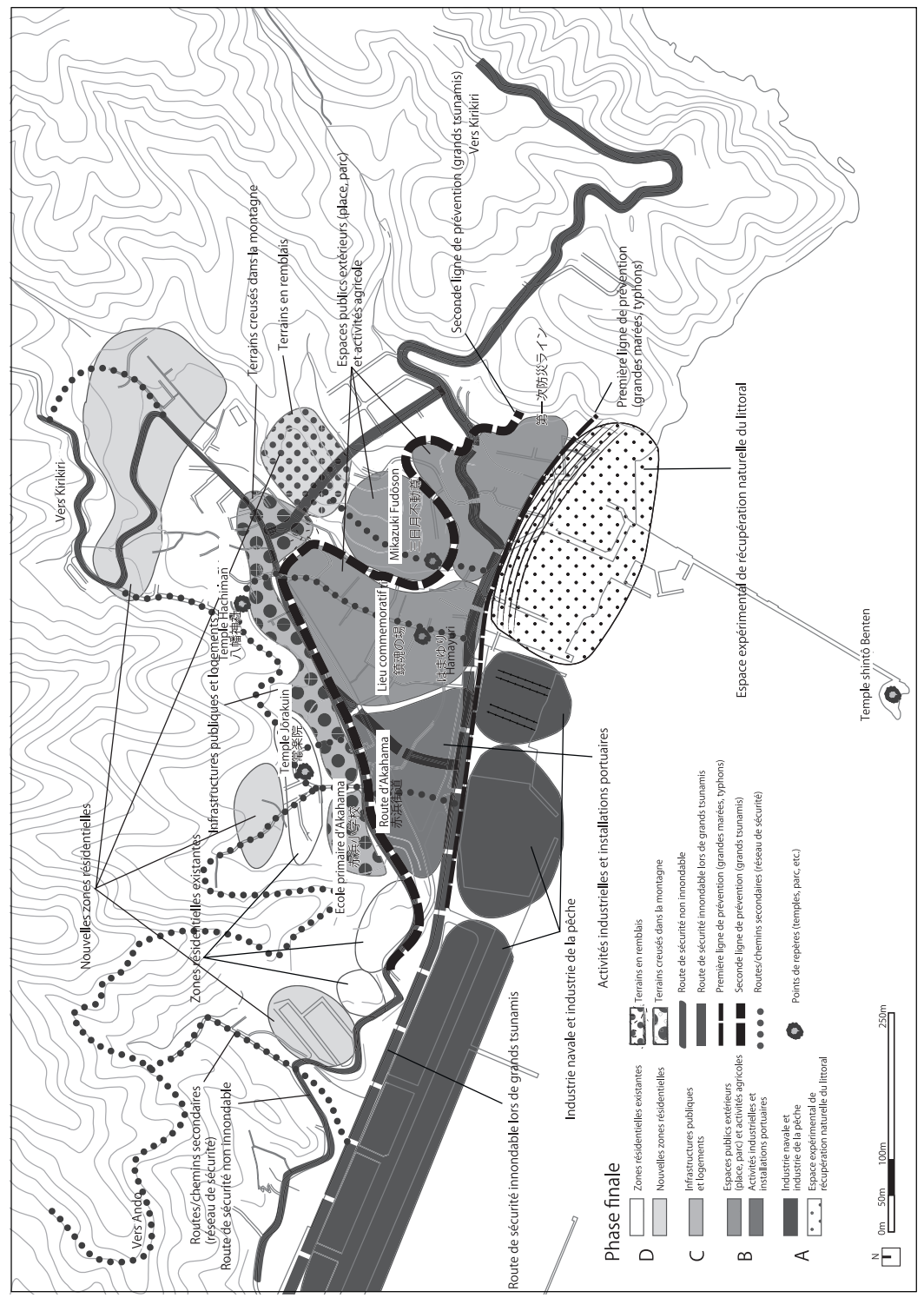

Fig. 4 Carte du projet de reconstruction proposé par le laboratoire de design urbain de l'université de Tokyo. 


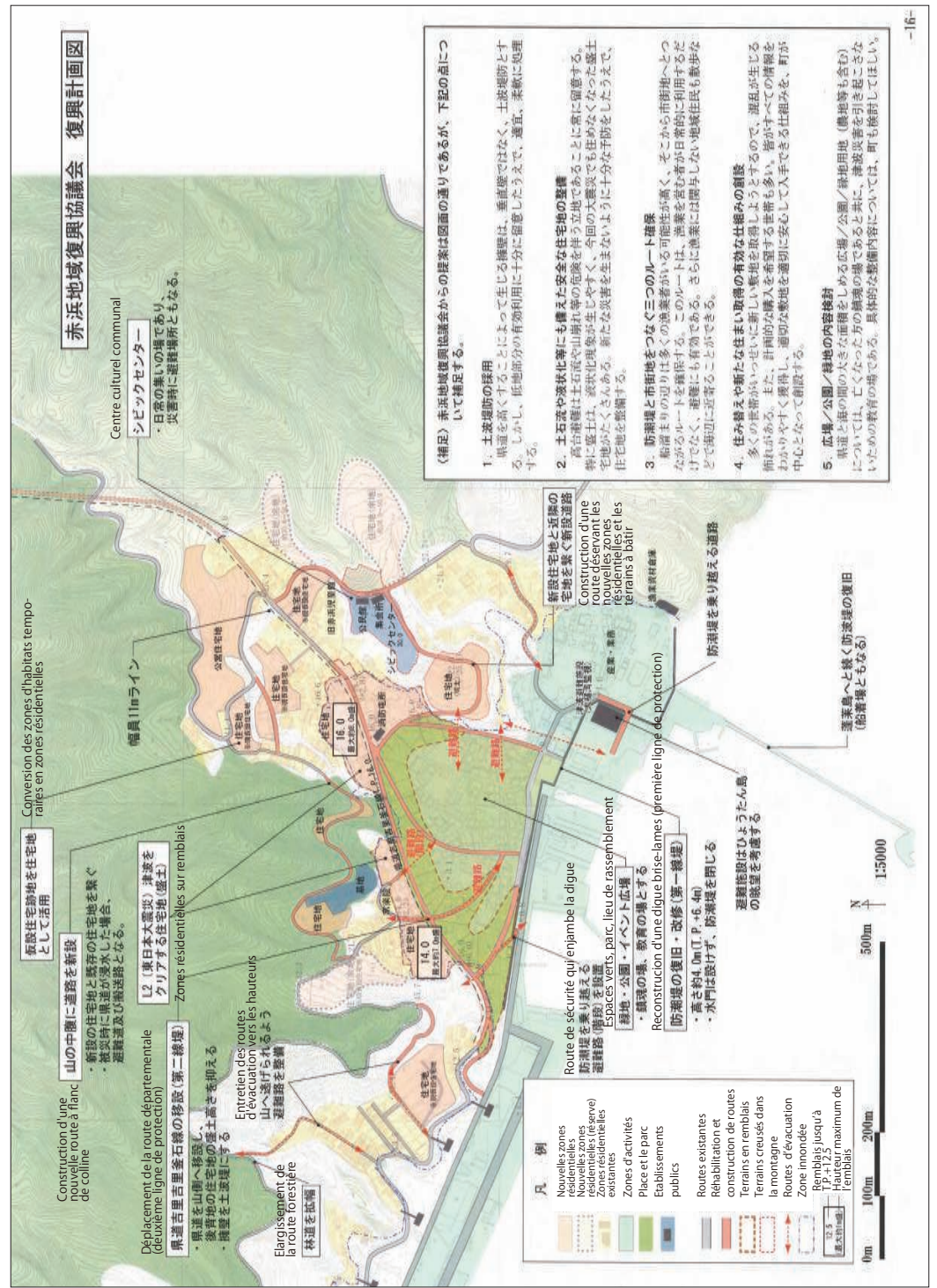

Fig. 5 Plan de reconstruction du quartier d'Akahama par le conseil municipal. Extrait d'Ōtsuchi-chō chiiki fukkō kyōgikai zentai kaigi shiryō 大槌町地域復興協議会全体会議資料 (Document de la réunion générale du Conseil municipal pour la reconstruction de la commune d'Ōtsuchi), p. 16. 
proposition. Nous avons, à plusieurs occasions, parlé du plan de reconstruction avec le président de l'association, M. Kawaguchi Hiromi 川口博美, ancien fonctionnaire de la ville. Le nouveau maire, M. Ikarigawa Yutaka 碇 川豊, a déclaré qu’il prendrait en considération les avis des habitants dans le plan de reconstruction. La première réunion du Comité de concertation pour la reconstruction de la commune d'Ōtsuchi (Ōtsuchi-chō chiiki fukkō kyōgikai 大槌町地域復興協議会) a eu lieu le 10 octobre. La commission des habitants a présenté le 26 octobre une "Proposition de plan de reconstruction après le séisme du quartier d'Akahama " (Akahama chiku shinsai fukkō keikakuan 赤浜地区震災復興計画案) lors de la deuxième réunion du Comité de concertation pour la reconstruction. Ce plan est en grande partie basé sur nos propositions dont ils ont pris connaissance. En particulier, la commission des habitants demandait le déplacement des maisons sur les hauteurs, l'installation d'un parc à l'endroit où se trouvait le bateau Hamayuri, le recours aux énergies renouvelables et l'installation d'une deuxième ligne de protection devant le quartier résidentiel déménagé en hauteur (fig. 5). Le 26 décembre, la commune a adopté le "Plan de reconstruction après le séisme de la commune d'Ōtsuchi " (Ōtsuchi-chō shinsai fukkō keikaku 大桘町震災復興計画) en se fondant sur cette proposition des habitants (Ōtsuchi-chō 2012). 


\section{Bibliographie}

Meiji daigaku rikō gakubu kenchiku gakka kenchiku shi kenchiku ron kenkyū shitsu 明治大学理工学部建築学科 建築史·建築論研究室 «Ōtsuchi (Iwate-ken Kamihei-gun Ōtsuchi-chō) »大槌(岩手県上閉伊郡大 槌町) (Ōtsuchi [ville d'Ōtsuchi, canton de Kamihei, département d'Iwate]), in Sanriku kaigan no shūraku, saigai to saisei :1896, 1933, 1960 三陸海岸の集落 一一災害亡再生:1896、1933、1960 (Les villages de la côte de Sanriku, désastres et reconstructions : 1896, 1933, 1960). En ligne : <http://d.hatena.ne.jp/meijikenchikushi/19760427/p1> (consulté le 27/11/2011).
Ōtsuchi-chō,

Saigai fukkō shitsu 大槌町災害復興室 (Ville d'Ōtsuchi, Cellule pour la reconstruction après les désastres) En ligne: <http://otsuchi.web.fc2.com/ new/main/page_saigaifukkousitu.html> (consulté le 09/02/2012).

\section{Toshi keikaku isan kenkyū kai}

都市計画遺産研究会 «Ōtsuchi-chō» 大槌町 (ville d'Ōtsuchi), in Sanriku kaigan no toshi keikaku. Fukkō keikaku shi ākaibu 三陸海岸の都市計 画/復興計画史アーカイブ (Urbanisme de la côte de Sanriku. Archives de I'histoire des plans de reconstruction). En ligne : <http://www45.atwiki.jp/ sanrikuplanning/pages/24.html>, (consulté le 27/11/2011). 